\title{
CHEMICAL VARIATION OF FIVE NATURAL EXTRACTS BY NON-POLAR SOLVENT
}

\author{
T.A. Prayitno ${ }^{1, \star}$, R. Widyorini ${ }^{1}$, G. Lukmandaru ${ }^{1}$
}

\begin{abstract}
Chemical compounds of wood preservation from plants vary and are not known specific to the species. Chemical analysis of plants is responsible to ensure active compound in natural extracts wood treatment. There are many sources of natural extracts found in Indonesia that were explored for wood preservatives chemicals. They are bark of acacia and alstonia, leaves of orthosiphon and azardirachta and Dioscorea tubers. The present study was aimed at investigating the variation of the chemical constituent of natural extracts material of wood preservative through GC-MS analysis. Five natural extract sources were acacia bark (Acacia spp.), pulai bark (Alstonia scholaris), kumis kucing leaves (Orthosiphon spp.), mimba leaves (Azardirachta indica), and gadung tubers (Dioscorea spp.). Two non-polar solvents, i.e., n-hexane and petroleum ether were used for five natural source extractions following ASTM soxhlet extraction. The research showed that triterpene and fatty acid derivatives were the major compounds present in five natural extracts. They were lupeol; 7,22-Ergostadienone; Lup-20(29)-en-3-one; Lup-20(29)-en-3-ol, acetate, (3.beta.)-; urs-12-en-3-one; ethanol,2,2-diethoxy-; stigmasta-5,22-dien-3-ol, acetate,(3.beta.)-; 5H-3,5a-Epoxynaphth(2,1-c)oxepin, dodecahydro-3,8,8,11a-tetramethyl-; linoleic acid; naphthalene, 1-methyl-. These compounds have been assigned as the possibly responsible to against termites or fungi.
\end{abstract}

Keywords: Acacia spp., Alstonia scholaris, Azardiachta indica, chemical compounds, Dioscorea spp., Orthosiphon spp., wood preservation.

\section{INTRODUCTION}

The new paradigm of wood preservation is related to the environmental safety. The use of synthetic preservative has long resulted in environs losses. Several synthetic chemicals have been banned recently for wood protection uses by the U.S. Environmental Protection Agency (EPA). Therefore, the search for natural, safe, friendly, and none non-polluting bioactive chemical compounds from plants as an alternative to synthetic preservative becomes essential (Hu et al. 2015).

Plant extract contains chemical constituent which has high potency for wood preservatives. Sources of plant extract are found many type such as leaves, barks, wood, tubers, seeds etc. The type of solvent to be used for plant extraction are found many type such as cold water, hot water, ethanol, benzene, ether and n-hexane. Many research have been conducted on natural plant extracts. Extractives of white mulberry heartwood were contained higher hydrocarbons, fatty acids, sterols, and phenols that were toxic against decay termites (Se Golpayegani et al. 2014). Fatty acid extracts from Acacia mollissima, Schinopsis lorentzii and Pinus brutia bark were indicated having an insecticide characteristic (Sen et al. 2017). The type of solvent has affected the extraction results and affected the resistance of termites (Kadir et al. 2015, Syofuna et al. 2012). N-hexane fraction from T. officinale leaves was detected and reported as antioxidant and antimicrobial (Ivanov et al. 2017). Prayitno et al. (2017) concluded that natural extracts have influenced the wood adhesion.

Acacia spp. belongs to the family Leguminosae and subfamily Mimosoideae. Acacia has been planted in more than eighty countries around the world and becomes prominent in tropical and subtropical regions of Asia, Africa, Central, and South America (Old et al. 2002). Extract of three species from Acacia leaf has po- 
tential in the development of natural food preservatives (Cock 2017). The heartwood of $A$. confusa possesses excellent decay resistance properties (Chen et al. 2013). A. mollissima bark extract could be used as alternative wood preservatives against Reticulitermes grassei termite (Tascioglu et al. 2012).

Orthosiphon plants are herbaceous shrubs from Lamiaceae family. Singh et al. (2015) reviewed that the plant has photochemical, pharmacological, and toxicological properties. Orthosiphon extracted with ethanol showed the highest efficiency to be anti-termite with mortality of termite 65\% (Aziz et al. 2013).

Alstonia scholaris is tropical tree belongs to Apocynaceae family. A. scholaris stem bark has anti-inflammatory activity on methanolic extract (Subraya et al. 2012). Extract of $A$. scholaris leaf has exhibited the activity of causing mortality in termite (Ahmed et al. 2011). Padding application of A. scholaris extract on teakwood block test gives highest adhesion strength (Prayitno and Widyorini 2016).

Azadirachta indica is an evergreen tree native to Southeast Asia. The tree belongs to Meliaceae family and has commercial name neem tree. It has been used medicinally for centuries because of its bioactive compounds. A. indica bark extracts could be employed to control fungal stains and molds on easily attacked freshly harvested logs (Antawi-Boasiako and Damoah 2010). Neem seed extract (azadirachtin) offer considerable protection to wood (Ssemaganda et al. 2011). Combination neem extract and chlorpyriphos could be efficiently utilized for termite control (Sotannde et al. 2011)

Dioscorea hipsida is a member of Dioscoreacea family. The plant produces tubers that contain toxic alkaloid (Dioscorides). The dioscorine within the tuber's starch would protect the coated materials from rotting by bacteria or fungi activity (Azman et al. 2015). Ragasa et al. (2016) reported the dichloromethane extracts of Dioscorea luzonensis Schauer were alkyl trans-ferulates ; $\beta$-sitosterol and ursolic acid. They were polyphenolic compound group. Kumar et al. (2017) reviewed the ethnopharmacological values and traditional use of Dioscorea spp. Savi et al. (2018) showed the potential antioxidant activity of the polysaccharide extracted from Dioscorea bulbifera. They suggested to analyze it by DPPH, ABTS, FRAP, OH radical removal, H2O2 removal and reducing power.

Chemical analysis of natural wood preservation is responsible for the development of wood preservative material. GC-MS is the best technique to identify the bioactive constituents of long chain hydrocarbons, alcohols, acids, esters, alkaloids, steroids, amino and nitro compounds, etc. (Karuppasamy et al. 2012). The combination of the best separation technique (GC) with the best identification technique (MS) made GC-MS an ideal method for qualitative analysis for volatile and semi-volatile bioactive compounds (Grover and Patni 2013). The objective of the present study was to know the variation of the chemical constituent of five natural extract sources potential for preservative material. Furthermore, the isolated matters produced by plants can be used as active principles in natural extracts wood treatment.

\section{MATERIALS AND METHODS}

\section{Materials}

Natural preservatives plant sources namely, akasia bark (Acacia spp.), pulai bark (Alstonia scholaris), kumis kucing leaves (Orthosiphon spp.), mimba leaves (Azardiachta indica), and gadung tubers (Dioscorea spp.) were collected from Yogyakarta, Indonesia. The site has a temperature range of $26-30^{\circ} \mathrm{C}$ and humidity range of $90 \%$. Two non-polar solvents were n-hexane and petroleum ether.

\section{Extraction procedure}

Five plant materials were air dried, grinded, and sieved by 40 mesh. Each dry plant sample was extracted with n- hexane and petroleum ether according to ASTM D1108-96 (2007). Two grams plant sample was placed in soxhlet extraction apparatus with $300 \mathrm{cc}$ of solvent. 


\section{GC/ MS analysis}

Separation and identification were performed on a GC/MS QP 2010 (Shimadzu) with an RTx-5MS column, $30 \mathrm{~m}$ (Restek Corp.) Helium was used as the carrier gas. The injection of $1 \mu \mathrm{l}(1 \mathrm{mg} / \mathrm{ml})$ was made in a split less mode using electron impact ionization (EI, $70 \mathrm{eV}$ ). The injector and detector temperature were $2500^{\circ} \mathrm{C}$. The program started at $500^{\circ} \mathrm{C}$ for 5 minutes, followed by an increase of $40^{\circ} \mathrm{C}$ min until $1200^{\circ} \mathrm{C}$ and remaining at this temperature for 1 minutes. Then, the temperature was set to $3000^{\circ} \mathrm{C}$ at $60^{\circ} \mathrm{C} / \mathrm{min}$ and maintained for 15 minutes. Compounds were identified by comparison of the mass spectra with those in the NIST 147 libraries. The extractive composition was determined by peak area integration.

\section{RESULTS AND DISCUSSION}

\section{Chemical component of five natural extracts}

In the present study, the constituents of five plant extracts were successively extracted by non-polar organic solvents of n-hexane and petroleum ether. The photochemical screening of Acacia spp. showed twenty peaks (Figure 1). The acacia bark extract with n-hexane showed GC peaks at retention times of 23,68 min and 32,3646,07 min. The acacia bark extract with petroleum ether showed GC peaks at retention times of 10,59-16,86 $\mathrm{min}, 23,68 \mathrm{~min}$, and 34,98-46,04 min. The compounds separated at 10,5-16,86 $\mathrm{min}$ in petroleum ether extract contained mainly hydrocarbon derivatives.
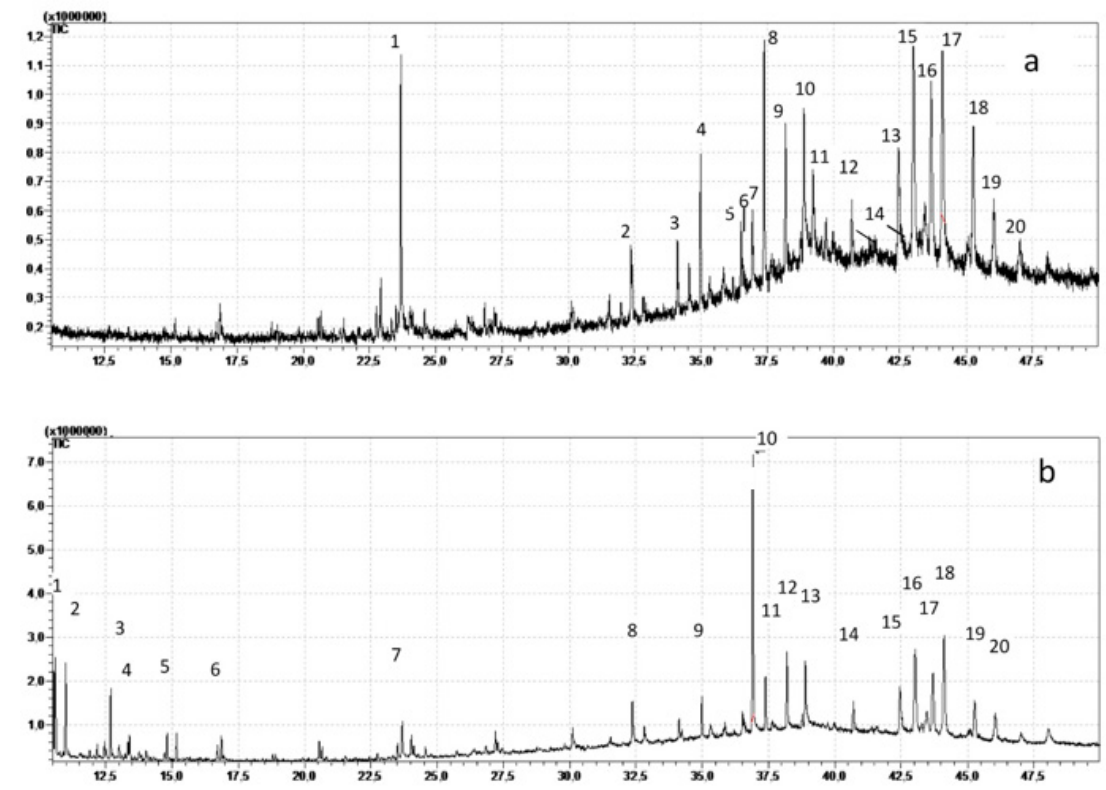

Figure 1: Chromatogram of organic compounds obtain from Acacia spp. bark in (a) n-hexane extract (b) petroleum ether extract.

(a) n-hexane extract: Dibutyl phthalate $(23,68 \mathrm{~min})^{1}, 1,2$-Benzenedicarboxylic acid, diisooctyl ester(32,36min $)^{2}$, Tetracosane, 11-decyl-(34,11 min $)^{3}$, 22-Tricosenoic acid $(34,98 \mathrm{~min})^{4}$, Nonacosane $(36,5 \mathrm{~min})^{5}$, Pentadecanal- $(36,58 \mathrm{~min})^{6}$, Hexacosanoic acid, methyl ester $(36,93 \mathrm{~min})^{7}$, Erucic acid $(37,37 \mathrm{~min})^{8}$, Octadecanal $(38,18 \mathrm{~min})^{9}, 13$-Tetradecen-1-ol acetate $(38,88 \mathrm{~min})^{10}$, Octacosanoic acid, methyl ester(39,22min $)^{11}$, Octadecanal(40,69min) ${ }^{12}$, Chondrillasterol(42,46min $)^{13}$, 1-Methyl-3,6-diazahomoadamantan-9-one thiosemicarbazone $(42,61 \mathrm{~min})^{14}, 7,22$-Ergostadienone $(43,01 \mathrm{~min})^{15}$, Lup-20(29)-en-3-one $(43,68 \mathrm{~min})^{16}$, Lupeol $(44,11 \mathrm{~min})^{17}$, Germanicol $(45,27 \mathrm{~min})^{18}, \mathrm{Lu}-$ $\operatorname{peol}(46,06 \mathrm{~min})^{19}, 7$-Hydroxy-6-methyl-oct-3-enoic acid $(46,07 \mathrm{~min})^{20}$

(b) petroleum ether extract : Naphthalene, 1-methyl-(10,59min $)^{1}$, Naphthalene, 1-methyl-(10,98min $)^{2}$, Tetradecane $(12,69 \mathrm{~min})^{3}$, Naphthalene, 2,6-dimethyl-(13,4min $)^{4}$, Dodecane, 2-methyl-6-propyl-(14,82min $)^{5}$, Octadecane $(16,86 \mathrm{~min})^{6}$, Dibutyl phthalate $(23,68 \mathrm{~min})^{7}$, 1,2-Benzenedicarboxylic acid, mono(2-ethylhexyl) ester(32,36min $)^{8}$, Erucic acid(34,98min) ${ }^{9}$, Oxirane, 2,2'-((1-methylethylidene) bis(4,1-phenyleneoxymethylene))bis-(36,9min $)^{10}, 22$-Tricosenoic acid $(37,37 \mathrm{~min})^{11}$, Octadecanal $(38,18 \mathrm{~min})^{12}, 17-$ Pentatriacontene $(38,88 \mathrm{~min})^{13}$, Octadecane, 1-(ethenyloxy)-(40,69min) ${ }^{14}$, Chondrillasterol $(42,45 \mathrm{~min})^{15}$, 7,22-Ergostadienone $(43,02 \mathrm{~min})^{16}$, Lup20(29)-en-3-one(43,7min) ${ }^{17}$, Lupeol(44,11 min $)^{18}$, Germanicol(45,26min $)^{19}, 7$-Hydroxy-6-methyl-oct-3-enoic acid $(46,04 \mathrm{~min})^{20}$ 
The data indicate that chemical component of acacia bark that solved with n-hexane was dominated with lupeol from triterpene derivatives, ergostandienon (7,22-ergostadienone) and germanicol (Figure 1). Another chemical is erucic acid from the fatty acid. Brassica rapa contained a high concentration of erucic acid and had toxic properties (Harborne 1973).

Acacia bark contains lupeol in both n-hexane and petroleum ether extract. Lupeol is the highest compound in n-hexane extract (16,52\%). Lupeol also found in the stem bark of Acacia mellifera and Acacia visco, and its antimicrobial and anti-inflammatory activities have been already demonstrated (Mutai et al. 2009, Pedemera et al. 2010). Lupeol is the pentacyclic triterpenes belonging to the lupane family.

Oxirane, 2,2'-((1-methylethylidene)bis(4,1-phenyleneoxymethylene))bis- is the highest compound of Acacia bark in petroleum ether extract. Oxirane is epoxide derivative that has a function as an adhesive (Ramalakshmi and Muthuchelian 2011). A minor compound present in Acacia spp. bark such as aromatic carbonyls (1,2-Benzenedicarboxylic acid, mono(2-ethylhexyl) ester). 1,2-Benzenedicarboxylic acid, (2-ethylhexyl) ester have antifungal, antitumor, anti-diabetic, anti-cancer, antioxidant, anti-inflammatory, antimicrobial (Syeda et al. 2011).
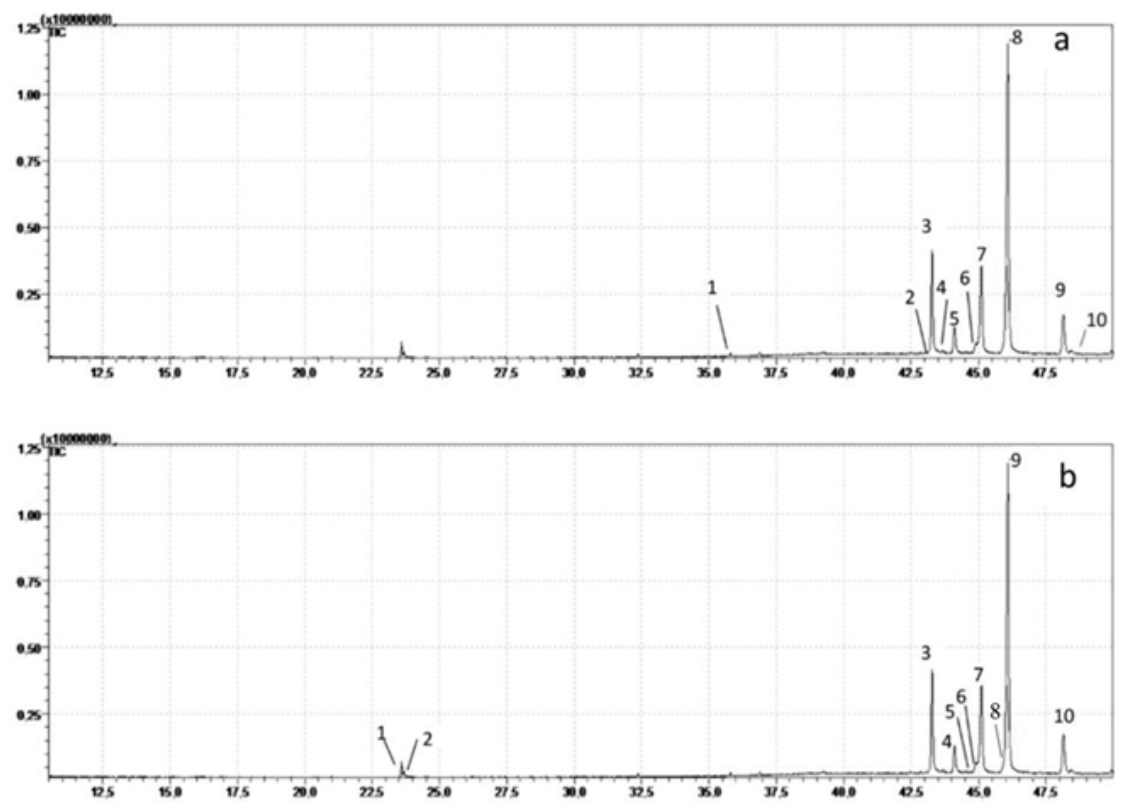

Figure 2: Chromatogram of organic compounds obtain from pulai bark in (a) n-hexane extract (b) petroleum ether extract.

(a) n-hexane extract: (all-E)-2,6,10,15,19,23-hexamethyl-2,6,10,14,18,22-tetracosahexaene (35.7 min)1 ${ }^{1}$ 9, 19-Cyclolanost-24-en-3-ol, (3.beta.)- (43.1 min $)^{2}$; urs-12-en-3-one (43,3 min) ${ }^{3}$; D-Norandrostan-16-ol, acetate, (5.alpha., 16.beta.)- (43,6 min) ${ }^{4}$; Lupeol (44,1 min) ${ }^{5}$; Lanosta-8,24-dien-3-ol, acetate, (3.beta.)- (44,9 min) ${ }^{6}$; 12-Oleanen-3-yl acetate, (3.alpha.)- (45,1 min) ${ }^{7}$; Lup-20(29)-en-3-ol, acetate, (3.beta.)-; Lup-20(29)-en-3-ol, acetate, (3.beta.)- $(46,2 \mathrm{~min})^{8}(48,2 \mathrm{~min})^{9}(48,8 \mathrm{~min})^{10}$.

(b) petroleum ether extract: Dibutyl phthalate $(23,5)^{1} ; 1,2$-Benzenedicarboxylic acid, bis(2-methylpropyl) ester (23,6 min $)^{2}$; urs-12-en-3one $(43,2)^{3}$; Lupeol $(44,1)^{4}$; Lanosta-8,24-dien-3-ol, acetate, (3.beta.)- $(44,8)^{5}$; Lup-20(29)-en-3-ol, acetate, (3.beta.)- (44,9 min) ${ }^{6}$; urs12-en-3-one (45,1 min $)^{7}$; trans-Biformene (45,9 min $)^{8} ; 5 \mathrm{H}-3,5 \mathrm{a}-$ Epoxynaphth $(2,1-\mathrm{c})$ oxepin, dodecahydro-3,8,8,11a-tetramethyl-(46,0 $\min )^{9}$; Lup-20(29)-en-3-ol, acetate, (3.beta.)- $(48,1 \mathrm{~min})^{10}$

The GC-MS analysis of pulai bark revealed that triterpene (Lup-20(29)-en-3-ol, acetate, (3.beta.)-(61,76\%) is a primary compound of pulai bark in n-hexane extract (Figure 2). This extract also found in Ficus carica leaves that showed potent and persistent irritant effects (Saeed and Sabir 2002).

The dominant compound of pulai bark in petroleum ether were 5H-3,5a-Epoxynaphth(2,1-c)oxepin, dodecahydro-3,8,8,11a-tetramethyl- (56,07\%). It was cyclic ethers that used for fragrance agents (Surburg and Panten 2006). Another compound, Lanosta-8, 24-dien-3-ol, acetate, (3.beta.)- have been reported to have an anti-amylase inhibitor, antimicrobial, antidiabetic properties (Arora and Meena 2016). 

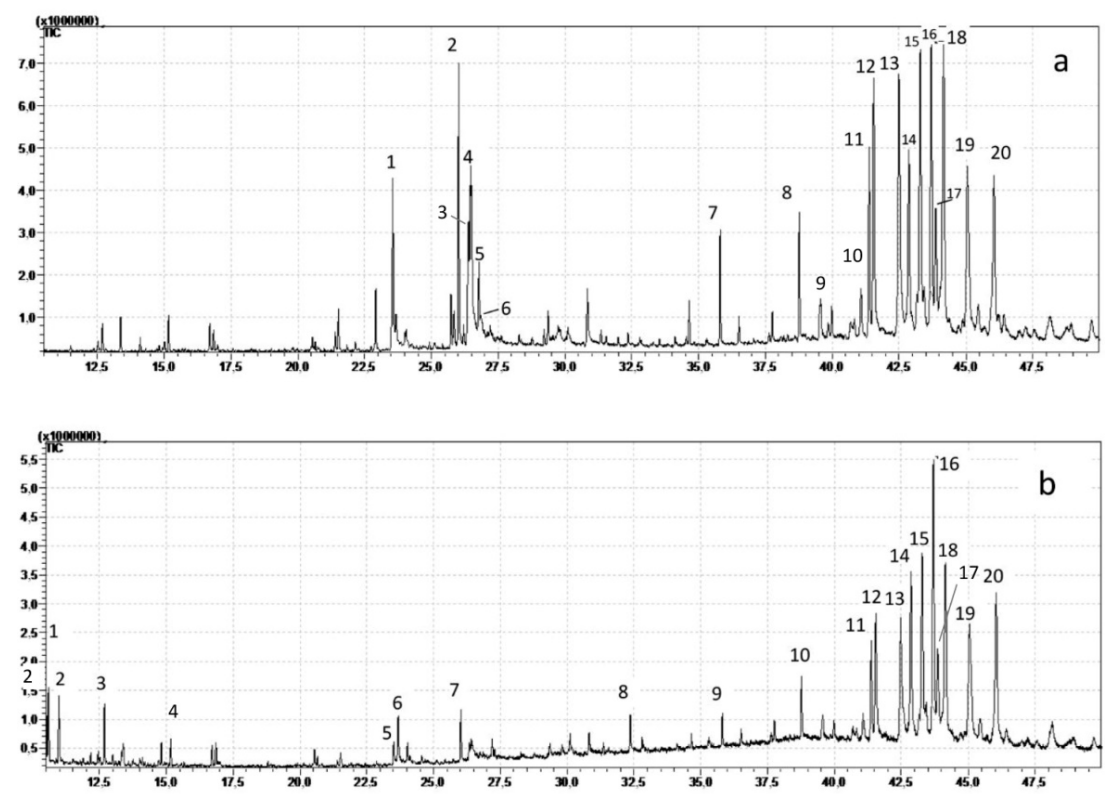

Figure 3: Chromatogram of organic compounds obtain from kumis kucing leaves in (a) n-hexane extract (b) petroleum ether extract.

(a) n-hexane extract : n-Hexadecanoic acid (23,561 min $)^{1} ; 3$ 3,7,11,15-Tetramethyl-2-hexadecen-1-ol (26,022 min $)^{2}$; 9,12-Octadecadienoic acid, methyl ester $(26,389 \mathrm{~min})^{3}$; Oleic Acid $(26,468 \mathrm{~min})^{4} ; 11,14,17$-Eicosatrienoic acid, methyl ester $(26,5 \mathrm{~min})^{5} ;$ Octadecanoic acid $(26,775 \mathrm{~min})^{6}$; (all-E)-2,6,10,15,19,23-hexamethyl-2,6,10,14,18,22-tetracosahexaene $(35,803 \mathrm{~min})^{7}$; Tetratetracontane $(38,762 \mathrm{~min})^{8}$; Vitamin E acetate (39,564 min ${ }^{9}$; Ergost-5-en-3-ol, (3.beta.)- $(41,071 \mathrm{~min})^{10}$; Tetratetracontane $(41,38 \mathrm{~min})^{11}$; Stigmasta-5,22-dien-3-ol, acetate, (3.beta.)- $(41,547 \mathrm{~min})^{12}$; gamma.-Sitosterol $(42,495 \mathrm{~min})^{13}$; urs-12-en-3-one $(42,861 \mathrm{~min}){ }^{14}$; urs-12-en-3-one $(43,292 \mathrm{~min})^{15}$; urs-12-en-3-one (43,701 min) ${ }^{16}$; 4,22-Cholestadien-3-one (43,872 min) ${ }^{17}$; urs-12-en-3-one (44,161 min) ${ }^{18}$; Stigmast-4-en-3-one (45,056 $\min )^{19}$; Urs-12-en-3-ol, acetate, (3.beta.)- $(46,041 \mathrm{~min})^{20}$

(b) petroleum ether extract : Naphthalene, 1-methyl- $(10,5 \mathrm{~min})^{1}$; Naphthalene, 1-methyl- $(10,9 \mathrm{~min})^{2}$; Tetradecane $(12,6 \text { min })^{3}$; Phenol, 2,4-bis(1,1-dimethylethyl)- (15,1 min $)^{4}$; n-Hexadecanoic acid (23,5 min $)^{5}$; Dibutyl phthalate $(23,6 \mathrm{~min})^{6} ; 3,7,11,15$-Tetramethyl-2-hexadecen-1-ol (26,0 min) ${ }^{7}$; 1,2-Benzenedicarboxylic acid, mono(2-ethylhexyl) ester (32,3 min $)^{8}$; (all-E)-2,6,10,15,19,23-hexamethyl-2,6,10,14,18,22-tetracosahexaene $(35,8 \mathrm{~min})^{9}$; Hentriacontane $(38,7 \mathrm{~min})^{10}$; Tetratetracontane $(41,3 \mathrm{~min})^{11}$; Stigmasta-5,22-dien-3-ol, acetate, (3.beta.)- $(41,5 \mathrm{~min})^{12}$; gamma.-Sitosterol $(42,4 \mathrm{~min})^{13}$; urs-12-en-3-one $(42,8 \mathrm{~min})^{15}$; urs-12-en-3-one $(43,2 \mathrm{~min})^{16} ;$ urs-12-en3 -one $(43,6 \mathrm{~min})^{16} ;$ 4,22-Stigmastadiene-3-one $(43,8 \mathrm{~min})^{17}$; urs-12-en-3-one $(44,1 \mathrm{~min})^{18}$; Stigmast-4-en-3-one $(45,0)^{19}$; Urs-12-en-3-ol, acetate, (3.beta.)- $(46,0 \mathrm{~min})^{20}$

Orthosiphon plant is herbaceus that has pharmological properties and mostly uses as medicinal. The primary compound identified in Orthosiphon leaves in n-hexane, and petroleum ether extract is urs-12-en-3-one from the triterpenoid group (Figure 3). This compound also found in Inula japonica that used to be acaricidar compounds (Duan et al. 2012). Acaricidar is pesticides that kill the member of arachnid subclass acari, which includes ticks and mites.

Several compounds of kumis kucing leaves that identified in both n-hexane and petroleum ether are Urs12-en-3-ol, acetate, (3.beta.)-, Stigmasta-5,22-dien-3-ol, acetate, (3.beta.)-, .gamma.-Sitosterol, Tetratetracontane, and n-Hexadecanoic acid. Bioactive component of Cynodon Dactylon revealed that n-hexadecanoic is used for pesticide and nematicide and Stigmasta-5,22-dien-3-ol, acetate, (3.beta.)- also have antimicrobial activity (Jebastella and Reginald 2015). 

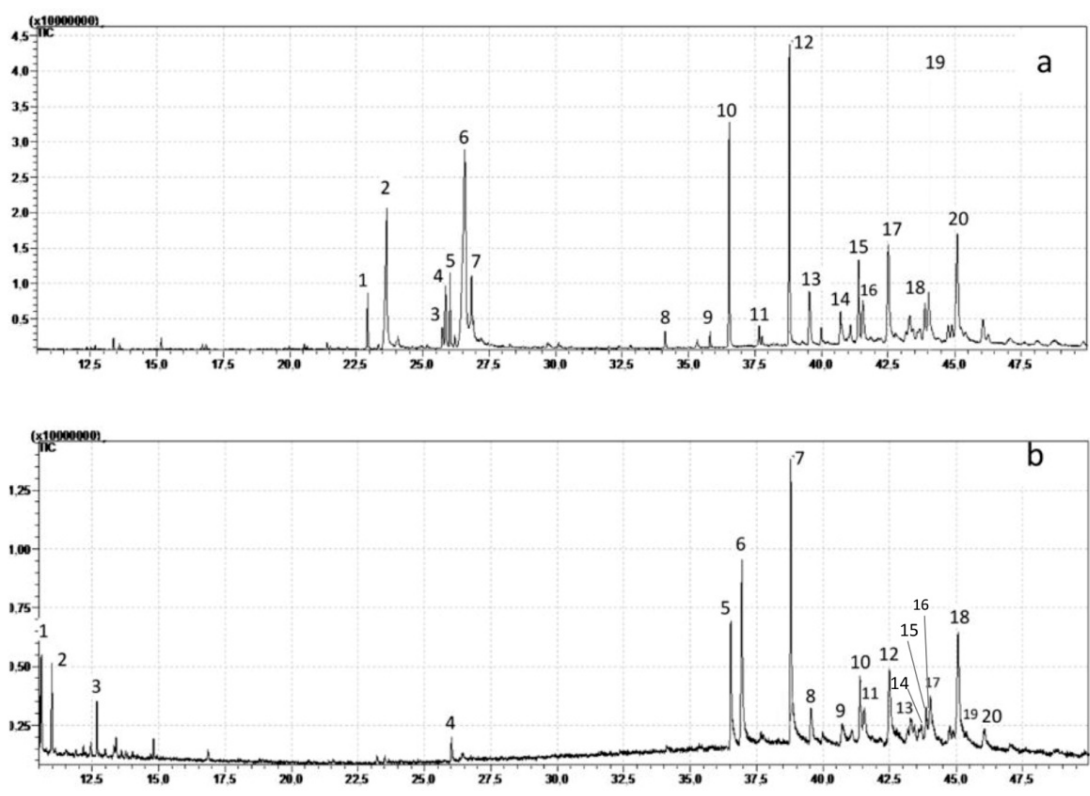

Figure 4: Chromatogram of organic compounds obtain from mimba leaves in (a) n-hexane extract (b) petroleum ether extract.

(a) n-hexane extract : 3,9-Dioxa-6-thiaundecane, 2,10-dimethyl- (22,9 min $)^{1} ; 3$-Ethylthio-1,2-propanediol (23,6 min $)^{2}$; Triallylphosphine $(25,7 \mathrm{~min})^{3}$; 4-Octadecenal $(25,8 \mathrm{~min})^{4}$; 1-Dimethyl(ethenyl)silyloxy-2-propene $(26,0 \mathrm{~min})^{5}$; Cyclohexanol, 1R-4cis-acetamido-5,6cis-epoxy-2trans,3cis-dimethoxy- $(26,5 \mathrm{~min})^{6}$; 1,3,4-Trimethoxy-butan-2-ol (26,8 min $)^{7}$; Ethanol, 2,2-diethoxy- $(34,1 \mathrm{~min})^{8}$; Silane, trimethyl((1-methylethylidene)cyclopropyl)- (35,8 min $)^{9}$; Ethanol, 2,2-diethoxy- $(36,5 \mathrm{~min})^{10}$; Ethanol, 2,2-diethoxy- $(37,6)^{11}$; Ethanol, 2,2-diethoxy- (38,7 min) ${ }^{12}$; 3-Amino-3-(4-amino-6-(dimethylamino)-1,3,5-triazin-2-yl)-1-propanol \# (39,5 min) ${ }^{13}$; Allyloxy-dimethyl-silane $(40,7 \mathrm{~min})^{14}$; Ethanol, 2,2-diethoxy- $(41,3 \mathrm{~min})^{15}$; 6-Ethoxy-6-methyl-2-cyclohexenone (41,5 min $)^{16}$; Allyloxy-dimethyl-silane (42,5 min) ${ }^{17}$; 6-Ethoxy-6-methyl-2-cyclohexenone (43,8 min) ${ }^{18}$; 1-Methyl-1-(prop-2-enyl)-1-silacyclopentane $(44,0 \text { min })^{19}$; Cobalt, (.eta.5-2,4-cyclopentadien-1-yl)((3,4-.eta.)-4,5-diethyl-1,2,2,3-tetramethyl-1-aza-2-sila-5-boracyclopent-3-ene-B5,N1)- (45,1 min) ${ }^{20}$.

(b) petroleum ether extract : 1H-Indole, 1,2-dimethyl-(10,593 $\mathrm{min})^{1}$; 1H-Indole, 2,6-dimethyl-(10,982 min $)^{2}$; Ethanol, 2,2-diethoxy$(12,687 \mathrm{~min})^{3}$; 1-Dimethyl(ethenyl)silyloxy-2-propene $(26,021 \mathrm{~min})^{4}$; Ethanol, 2,2-diethoxy- $(36,528 \mathrm{~min})^{5}$; 1-Cyclohexyldimethylsilyloxyoctadecane $(36,943 \mathrm{~min})^{6}$; Ethanol, 2,2-diethoxy- $(38,781 \mathrm{~min})^{7}$; L-Arabinopyranoside, 1-(benzothiazol-2-ylthio)-3,4-O-isopropylidene- $(39,541 \mathrm{~min})^{8}$; Methyl 2,5,6-tri-O-acetyl-3-acetamido-3-deoxy-D-altrofuranoside $(40,71 \mathrm{~min})^{9}$; Ethanol, 2,2-diethoxy- (41,387min) ${ }^{10}$; 2-(1-Hydroxy-9a,11a-dimethylhexadecahydrocyclopenta(a)phenanthren-1-yl)-2-methylpropionic acid, ethyl ester (41,548 $\mathrm{min})^{11} ; 2-\left(((2-M e t h o x y e t h o x y)\right.$ methyl)thio $)-1 \mathrm{H}$-imidazole $(41,585 \mathrm{~min})^{12}$; Isotridecyl alcohol, trimethylsilyl derivative(42,496 min) ${ }^{13}$; 1-Aza-2-sila-5-boracyclopent-3-ene, 4,5-diethyl-1,2,2-trimethyl-3-(1-methylethenyl)-( 43,28 min) ${ }^{14}$; Exo-norbornanol, methyl(pentamethylene)silyl ether(43,696 min) $)^{15}$; 1-Dimethyl(ethenyl)silyloxy-2-propene(43,878 min) ${ }^{16}$; 3-O-Methyl-d-glucose

(44,03 min ${ }^{17}$; 1-Dimethyl(ethenyl)silyloxy-2-propene(45,065 min) ${ }^{18}$; Methyl 2,3-diacetamido-2,3-dideoxy-.alpha.-D-glucopyrano$\operatorname{side}(45,374 \mathrm{~min})^{19} ;$ 1-Aza-2-sila-5-boracyclopent-3-ene, 4,5-diethyl-1,2,2-trimethyl-3-(1-methylethenyl)- $(46,07 \mathrm{~min})^{20}$

Mimba (A. indica) belongs to Meliaceae family is one of the most promising pesticides. The chemical compounds of seeds bark and leaves were isolated and identified as antiseptic, antiviral, antipyretic, anti-inflammatory, anti-ulcer and antifungal uses (Britto and Sheeba 2011, Chattopadhyay 1999). Mimba extracts alone or mixed with copper sulfate, and boric acid confirmed their antifungal activity was protecting Mangifera indica and Albizia saman woods (Islam et al. 2009).

The major compounds present in mimba leaf extract in n-hexane and petroleum ether were ethanol, 2,2-diethoxy-(Figure 4). It belongs to alcohol derivatives. According to Al-hashemi and Hossain (2016), alkaloids, steroids, flavonoids, tannins, saponins and amino acid were present in all polarities of crude neem leaf extracts except anthraquinone and triterpenoids. 

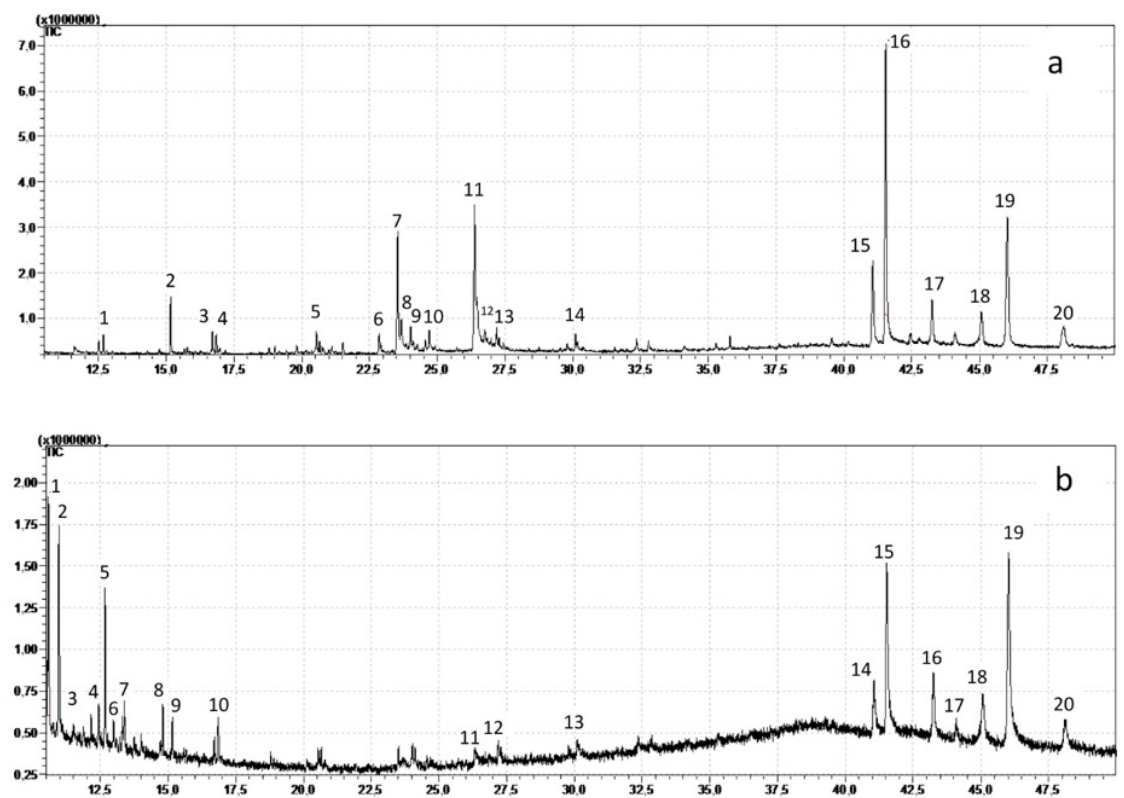

Figure 5: Chromatogram of organic compounds obtain from gadung tubers in (a) n-hexane extract (b) petroleum ether extract.

(a) n-hexane extract : Tetradecane(12,6 min $)^{1}$; Phenol, 2,4-bis(1,1-dimethylethyl)-(15,1 min)2 ; 3-Hexadecene, (Z)-(16,7 min) Eicosane $(16,8 \mathrm{~min})^{4} ;$ 1-Heptadecene(20,5 min $)^{5}$; 2-Isopropyl-5-methyl-1-heptanol(22,8 min ${ }^{6} ;$ n-Hexadecanoic acid(23,5 min $)^{7}$; Dibutyl phthalate $(23,6 \mathrm{~min})^{8} ; 1$-Tricosene $(24,0 \mathrm{~min})^{9}$; Eicosanoic acid $(24,7 \mathrm{~min})^{10} ; 9,12$-Octadecadienoic acid $(\mathrm{Z}, \mathrm{Z})$ - $(26,3 \mathrm{~min})^{11} ; 9,12,15-\mathrm{Oc}-$ tadecatrienoic acid, (Z,Z,Z)- $(26,4 \mathrm{~min})^{12}$; 1-Nonadecanol $(27,191 \mathrm{~min})^{13}$; 1-Tricosene $(30,103 \mathrm{~min})^{14}$; Campesterol $(41,057 \mathrm{~min})^{15}$; Stigmasta-5,22-dien-3-ol, acetate, (3.beta.)-(41,539 min ${ }^{16}$; urs-12-en-3-one $(43,241 \mathrm{~min})^{17}$; Olean-12-ene $(45,066 \mathrm{~min})^{18} ; 5 \mathrm{H}-3,5 \mathrm{a}-\mathrm{Ep}-$ oxynaphth(2,1-c)oxepin, dodecahydro-3,8,8,11a-tetramethyl- (46,02 min $)^{19}$; Lup-20(29)-en-3-ol, acetate, (3.beta.)-(48,099 min $)^{20}$.

(b) petroleum ether extract : Naphthalene, 1-methyl-(10,5 min $)^{1}$; Naphthalene, 1-methyl-(10,9 min $)^{2}$; Hexadecane, 2,6,10,14-tetramethyl-(12,17 min) ${ }^{3}$; Biphenyl (12,437 min $)^{4}$; Tetradecane (12,68 min $)^{5}$; Naphthalene, 1,7-dimethyl- $(13,317 \mathrm{~min})^{6}$; Naphthalene, 2,6-dimethyl- (13,394 min $)^{7}$; Pentadecane $(14,81 \mathrm{~min})^{8}$; Phenol, 2,6-bis(1,1-dimethylethyl)- (15,161 min $)^{9}$; Eicosane, 10-methyl- $(16,854 \text { min })^{10}$; 2-(4-Hydroxybutyl)cyclohexanol (26,333 min) ${ }^{11}$; Trifluoroacetic acid, n-heptadecyl ester (27,185 min) ${ }^{12}$; Pentadec-7-ene, 7-bromomethyl- (30,109 min) $)^{13}$; Ergost-7-en-3-ol, (3.beta.)- (41,049 min) ${ }^{14}$; Stigmasta-5,22-dien-3-ol, acetate, (3.beta.)- $(41,524 \text { min })^{15}$; urs-12-en-3one $(43,25 \mathrm{~min})^{16} ; 1,2,4$-Triazole, 4 -(N-(2-hydroxyethyl)-N-nitro)amino- $(44,095)^{17}$; Olean-12-ene $(45,056 \text { min })^{18}$; 5H-3,5a-Epoxynaphth(2,1-c)oxepin, dodecahydro-3,8,8,11a-tetramethyl- $(46,02)^{19}$; Longifolenaldehyde $(48,112 \mathrm{~min})^{20}$.

The dominant gadung tubers chemical constituents in n-hexane extract are steroid derivatives (Stigmasta5,22-dien-3-ol, acetate, (3.beta.), hydrocarbon (5H-3,5a-Epoxynaphth(2,1-c)oxepin,) and fatty acid (linoleic acid )(Figure 5). Another compound in Gadung tubers n-hexane extract was linoleic acid- (11,9\%) which has anti-inflammatory property. $\mathrm{N}$-Hexadecanoic acid $(8,96 \%)$ was palmitic acid, and it used to antimicrobial, hypocholesterolemic, nematicide, pesticide (Dr. Duke's databases). Hexadecanoic acid also the significant pyhtoconstituents of gadung in butanol extraction (Om et al. 2016).

The primary compounds present in gadung tubers extract in petroleum ether were Naphthalene, 1-methyl$(23,26 \%)$. Naphthalene produced by from Magnolia flowers can inhibit natural fungi from proliferating (Chen et al. 1998). Muscodor vitigenus fungus also produced naphthalene which purports to be insect repellents (Daisy et al. 2002). Naphthalene, 1-methyl- occur naturally in fossil fuels and produced commercially from either coal tar or petroleum, and used for making insecticide carbaryl, leather tanning agents, and dye intermediates (ATSDR 2005). 


\section{Variation of five natural extracts}

Type of solvent used have effects on the percentage of extraction result. Table 1 summarizes the total percentage extracts and the dominant compound in five plant extracts for the natural preservative. The highest extract percentage is found in pulai barks in both ether $(1,97 \%)$ and $n$-hexana solvent $(1,91 \%)$. The lowest extract is produced by gadung tubers in ether $(0,19 \%)$ and $n$-hexane $(0,22 \%)$. The comparison of the dominant compound of the extracts by different solvent gives a similar figure but different percentage. It is found petroleum ether solvent can detect the compound in lower retention time (Figure 1, Figure 2, Figure 3, Figure 4 and Figure 5). According to Efeovbokhan et al. (2015), petroleum ether solvent gave the highest oil yield from Moringa seed followed by hexane then isopropyl alcohol. The comparison of five extracts in terms of the identified compounds, derivatives, and the predicted activity are listed in Table 2. Triterpene derivatives were dominating in the five natural preservatives extract sources. Other compounds are the fatty acid, alcohol, steroid, hydrocarbon, and palmitic acid. It's clear that the most abundant chemical of five natural preservatives extracts by the non-polar solvent is belong to lipophilic constituents. The previous study reported that lipophilic extracts identified from the Pinus bark by GC and GC-MS were composed of four component families i.e., fatty acids and alcohols; monoterpenes and sesquiterpenes; resin acids; steroids and triterpenoids (Masendra et al. 2017). By GC-MS analyses, some potential bioactive compounds were detected for the development of natural preservatives from those 5 natural extracts. It is noticed that most of the detected lipophilic compounds here have not been assayed against wood degraders such as destroying-fungi, termites, and marine borers. Therefore three sets of work should be conducted in the near future, i.e., isolation and identification of bioactive compounds from the crude extracts; bioassay of isolated compounds, as well as impregnation and bioassay of bioactive extracts into the solid wood.

Table 1: Percentage extracts and dominant chemical compounds in five natural preservatives extract source.

\begin{tabular}{|c|c|c|c|c|c|c|c|c|c|c|}
\hline \multirow{2}{*}{$\begin{array}{l}\text { Percentage } \\
\text { extracts, } \\
\text { Dominant } \\
\text { Compound }\end{array}$} & \multicolumn{5}{|c|}{ N-hexane (\%) } & \multicolumn{5}{|c|}{ Petroleum ether (\%) } \\
\hline & Acacia & Pulai & $\begin{array}{l}\text { Kumis } \\
\text { kucing }\end{array}$ & Mimba & Gadung & Acacia & Pulai & $\begin{array}{l}\text { Kumis } \\
\text { kucing }\end{array}$ & Mimba & Gadung \\
\hline $\begin{array}{l}\text { Percentage } \\
\text { extracts }(\%)\end{array}$ & 0,46 & 1,91 & 0,93 & 0,40 & 0,22 & 0,82 & 1,97 & 0,36 & 0,35 & 0,19 \\
\hline Lupeol & 16,52 & 4,54 & & & & 11,34 & 4,02 & & & \\
\hline $\begin{array}{c}\text { 7,22- } \\
\text { Ergostadienone }\end{array}$ & 13,21 & & & & & 8,87 & & & & \\
\hline $\begin{array}{l}\text { Lup-20(29)-en- } \\
\text { 3-one }\end{array}$ & 10,08 & & & & 2,46 & 6,34 & 4,02 & & & \\
\hline $\begin{array}{l}\text { Lup-20(29)-en- } \\
\text { 3-ol, acetate, } \\
\text { (3.beta.)- }\end{array}$ & & 61,76 & & & 2,46 & & 0,89 & & & \\
\hline Urs-12-en-3-one & & 14,66 & 34,33 & & 4,07 & & 28,78 & 45,88 & & 5,25 \\
\hline $\begin{array}{l}\text { Ethanol, 2,2- } \\
\text { diethoxy- }\end{array}$ & & & & 28,32 & & & & & 35,39 & \\
\hline $\begin{array}{l}\text { Stigmasta-5,22- } \\
\text { dien-3-ol, } \\
\text { acetate, } \\
\text { (3.beta.)- }\end{array}$ & & & 7,85 & & 27,75 & & & 5,86 & & 14,58 \\
\hline $\begin{array}{c}\text { 5H-3,5a- } \\
\text { Epoxynaphth(2, } \\
\text { 1-c)oxepin, } \\
\text { dodecahydro- } \\
3,8,8,11 \mathrm{a}- \\
\text { tetramethyl- }\end{array}$ & & & & & 16,54 & & 56,07 & & & 22,16 \\
\hline Linoleic acid & & & 3,33 & & 11,99 & & & & & \\
\hline $\begin{array}{l}\text { Naphthalene, 1- } \\
\text { methyl- }\end{array}$ & & & & & & 11,97 & & 4,84 & & 23,26 \\
\hline $\begin{array}{c}\mathrm{N}- \\
\text { Hexadecanoic } \\
\text { acid }\end{array}$ & & & 3,73 & & 8,96 & & & 0,58 & & \\
\hline
\end{tabular}


Table 2: Chemical compounds activity of five natural preservatives extract source.

\begin{tabular}{|l|l|l|}
\hline \multicolumn{1}{|c|}{ Compound } & Derivatives & \multicolumn{1}{c|}{ Activity } \\
\hline Lupeol & Triterpene & Antimicrobial, antiinflammatory, \\
\hline 7,22-Ergostadienone & Triterpene & unknown \\
\hline Lup-20(29)-en-3-one & Triterpene & Antimalarial, anti-inflammatory ${ }^{3}$ \\
\hline Lup-20(29)-en-3-ol, acetate, (3.beta.)- & Triterpene & Anti-inflammatory \\
\hline urs-12-en-3-one & Triterpene & No activity reported ${ }^{3}$ \\
\hline Ethanol,2,2-diethoxy- & Alcohol & unknown \\
\hline $\begin{array}{l}\text { Stigmasta-5,22-dien-3-ol, acetate, } \\
\text { (3.beta.)- }\end{array}$ & Steroid & $\begin{array}{l}\text { Biomarker for the presence of } \\
\text { (marine) algal matter in the } \\
\text { environment }\end{array}$ \\
\hline $\begin{array}{l}\text { 5H-3,5a-Epoxynaphth(2,1-c)oxepin, } \\
\text { dodecahydro-3,8,8,11a-tetramethyl-, }\end{array}$ & Hydrocarbon & $\begin{array}{l}\text { Fragrance agents } \\
\text { 6racid }\end{array}$ \\
\hline linoleic acid & Fatty acid & $\begin{array}{l}\text { Anti-inflammatory, Nematicide, } \\
\text { Insectifuge, Hypocholesterolemic, } \\
\text { Cancer preventive, Hepatoprotective, } \\
\text { Antihistaminic, Antiacne, } \\
\text { Antiarthritic, Antieczemic }\end{array}$ \\
\hline Naphthalene, 1-methyl- & Hydrocarbon & $\begin{array}{l}\text { Insecticide carbaryl, leather tanning } \\
\text { agents, and dye intermediates }\end{array}$ \\
\hline N- Hexadecanoic acid & Palmitic acid & $\begin{array}{l}\text { Antimicrobial, hypocholesterolemic, } \\
\text { nematicide, pesticide }\end{array}$ \\
\hline
\end{tabular}

${ }^{1}$ Mutai et al. 2009, ${ }^{2}$ Pedemera et al. 2010, ${ }^{3}$ USDA 2016, ${ }^{4}$ Lucetti et al. 2010, ${ }^{5}$ Malik 2016, ${ }^{6}$ Surburg and Panten 2006, ${ }^{7}$ Anburaj et al . $2016,{ }^{8}$ ATSDR 2005

\section{CONCLUSIONS}

The results of this study shows that five plant sources extracted by n-hexane and petroleum ether gave several chemical compounds which have potential to be natural preservatives. They were Lupeol, 7,22-Ergostadienone, Lup-20(29)-en-3-one, Lup-20(29)-en-3-ol, acetate, (3.beta), Urs-12-en-3-one, Ethanol, 2,2-diethoxy-, Stigmasta-5,22-dien-3-ol, acetate,(3.beta.)-, 5H-3,5a-Epoxynaphth(2,1-c)oxepin,dodecahydro-3,8,8,11a-tetramethyl-, linoleic acid, Naphthalene, 1-methyl-, and N- Hexadecanoic acid. Moreover, these results could be useful in the research for isolating and evaluating the compounds against termites or fungi.

\section{ACKNOWLEDGEMENTS}

This research work was carried out within a research project funded by Directorate General of Higher Education, Ministry of Education and Culture, Republic of Indonesia. The project number is LPPM-UGM/1448/ LIT/2013. The authors would like to express their sincere appreciation for technical support from Nurmadina and Masendra.

\section{REFERENCES}

Ahmed, S.; Zafar, M.I.; Hussain A. Riaz, M.A.; Shahid, M. 2011. Evaluation of plant extracts on mortality and tunneling activities of subterranean termites in Pakistan. In: Stoytcheva M (ed) Pesticides in the Modern World - Pests Control and Pesticides Exposure and Toxicity Assessment. InTech: Rijeka, Croatia. ISBN: 978-953-307-457-3. https://doi.org/10.5772/18999

Al-Hashemi, Z.S.S.; Hossain, M.A. 2016. Biological activities of different neem leaf crude extracts used locally in Ayurvedic medicine. Pacific science review. Natural science and engineering 18:128-131. http://dx.doi.org/10.1016/j.psra.2016.09.013

Anburaj, G.; Marimuthu, M.; Rajasudha, V.; Manikandan, R. 2016. Photochemical screening and GC-MS analysis of ethanolic extract of Tecima stans (Family: Bignoniaceae) Yellow Bell Flowers. Journal of 
Pharmacognosy and Phytochemistry 5(4): 172-175. http://dx.doi.org/10.22271/phyto

Antwi-Boasiako, C.; Damoah, A. 2010. Investigation of synergistic effect of extracts from Erythrophleum suaveolens, Azadirachta indica, and Chromolaena odorata on the durability of Antiaris toxicaria. Int Biodeter Biodegr 64: 97-103. http://dx.doi.org/10.1016/j.ibiod.2009.08.005

Arora, A.; Meena, S. 2016. Qualitative premilinary phytochemical screening and GC-MS analysis of root of Sarcostemma viminale (L) R. BR., an endangered plant. International Journal of Pharmaceutical Research and Bio-science 5(2):89-100. http://www.ijprbs.com/index.php

Asghar, S.F.; Habib-Ur, Rehman.; Choudahry, M.I.; Atta-Ur-Rahman. 2011. Gas Chromatography-Mass Spectrometry (GC-MS) analysis of extract (oil) and bioassays of crude extract of Iris germanica. International Journal of Genetics and Molecular Biology 3(7): 95-100. http://www.academicjournals.org/ijgmb

ASTM. 2007. Ether solubility in wood. ASTM D-1108-96. 2007. Annual Book of ASTM Standard.

ATSDR. 2005. Toxicological profile for naphthalene, 1- methylnaphthalene, and 2-methylnaphthalene. Agency for Toxic Substances and Disease Registry. Atlanta, Georgia.

Aziz, A.; Prayitno, T.A.; Hadikusumo, S.A.; Santoso, M. 2013. Uji ekstrak etanol kumis kucing (Orthosiphon sp.) sebagai pengawet alami kayu. Jurnal Ilmu Kehutanan 7(1). 48-56. https://doi.org/10.22146/jik.6137

Azman, I.; Mutalib, S.A.; Yusoff, S.F.M.; Fazry, S.; Noordin, A.; Kumaran, M.; Lazim, A.M. 2015. Novel Dioscorea hipsida strach-based hydrogels and their beneficial use as disinfectants. Journal of Bioactive and Compatible Polymers 31(1): 42-59. https://doi.org/10.1177/0883911515597704

Britto, A.J.; Sheeba, D.H. 2011. Azadiracta indica juss - a potential antimicrobial agent. International Journal of Applied Biological and Pharmaceutical Technology: 4550-4557. http://dx.doi.org/10.1016/j.jtusci.2013.05.0031.

Chattopadhyay, R. 1999. Possible mechanism of anti-hyperglycemic effect of Azadirachta indica leaf extract. Journal of Ethnopharmacology 67 (3): 373-376. https://doi.org/10.1016/s0378-8741(99)00094-x

Chen, J.; Henderson, G.; Grimm, C.C.; Lloyd, S.W.; Laine, R.A. 1998. Termites fumigate their nests with naphthalene. Nature 392: 558-559. https://doi.org/10.1038/33305

Chen, P.; Chen, Y.; Yeh, T.; Chang, S. 2014. Mechanism of decay resistance of heartwood extracts from Acacia confusa against the brown-rot fungus Laetiporus sulphureus. Wood Sci Technology 8 (3):451-465. https://doi.org/10.1007/s00226-014-0615-6.

Cock, I.E. 2017. Australian Acacia spp. extracts as natural food preservatives: Growth inhibition of food spoilage and food poisoning bacteria. Pharmacognosy Communications 7(1): 4-15. https://doi.org/10.5530/pc.2017.1.2

Daisy, B.H.; Strobel, G.A.; Castillo, U.; Ezra, D.; Sears, J.; Weaver, D.K.; Runyon, J.B. 2002. Naphthalene, an insect repellent, is produced by Muscodor vitigenus, a novel endophytic fungus. Microbiology 148:3737-3741. https://doi.org/10.1099/00221287-148-11-3737

Duan, D.; Bu, C.; Ma, L.; Liu, Y.; Wang, Y.; Shi G. 2012. Identification of acarcidal compounds in inula japonica extracts against Tetranychus cinnabarinus. In: Zhu, E.; Sambath, S. (eds). Information Technology and Agricultural Engineering. Advances in Intelligent and Soft Computing134. Springer: Berlin, Heidelberg. https://doi.org/10.1007/978-3-642-27537-1_88

Efeovbokhan, V.E.; Hymore, F.K.; Raji , D.; Sanni, S.E. 2015. Alternative solvents for Moringa oleifera seeds extraction. Journal of Applied Sciences 15(8): 1073-1082. https://doi.org/10.3923/jas.2015.1073.1082

Grover, N.; Patni, V. 2013. Phytochemical characterization using various solvent extracts and GC-MS analysis of methanolic extract of Woodfordia fruticosa (L) Kurz. Leaves. Int J Phar \& Pharma Sci 5(4): 291295. http://ijppsjournal.com/index.htm 
Harborne, J.B. 1973. Phytochemical methods. Chapman and Hall. https://catalogue.nla.gov.au/Record/174917

Hu, J.B.; Chang, S.S.; Peng, K.Y.; Hu, K.F.; Thevenon, M. 2015. Bio-susceptibility of shells of Camellia oleifera Abel. fruits to fungi and termites. Int Biodeter Biodegr 104:219-223. https://doi.org/10.1016/j.ibiod.2015.06.011

Islam, M.; Shams, I.; Ilias, G.N.M.; Hannan, O. 2009. Protective antifungal effect of neem (Azadirachta indica) extracts on mango (Mangifera indica) and rain tree (Albizia saman) wood. Int Biodeter Biodegr 63(2): 241-243. https://doi.org/10.1016/j.ibiod.2008.07.010

Ivanov, I.; Petkova, N.; Tumbarski, J.; Dincheva, I.; Badjakov, I.; Denev, P.; Pavlov, A. 2018. GC-MS characterization of $\mathrm{n}$-hexane soluble fraction from dandelion (Taraxacum officinale Weber ex. F.H. Wigg.) aerial parts and its antioxidant and antimicrobial properties. Z Naturforsch C 73(1-2):41-47. https://doi.org/10.1515/znc-2017-0107.

Jebastella, J.; Reginald, A.M. 2015. Bioactive components of Cynodon dactylon using ethanol extract. World J Pharm Sci 3(12): 2388-2391. http://www.wjpsonline.org/

Kadir, R.; Awang, K.; Khamaruddin, Z.; Soit, Z. 2015. Chemical composition and termiticidal activities of the heartwood from Calopyllum inophyllum L. Anais da Academia Brasileira de Ciências 87(2): 743 751. http://dx.doi.org/10.1590/0001-3765201520140041

Karuppasamy, B.; Antony, N.; Veerabahu, R.M. 2012. GC-MS analysis of Polycarpaea corymbosa (L.) Lam whole plant. Asian Pacific Journal Tropical Biomedicine 1289-1292. https://doi.org/10.1016/S2221-1691(12)60402-X

Kumar,S.; Das,G.; Shin, H; Patra, J.K. 2017. Dioscorea spp. (A Wild Edible Tuber): A study on its ethnopharmacological potential and traditional use by the local people of Similipal Biosphere Reserve, India. Front Pharmacol 8: 52. https://doi.org/10.3389/fphar.2017.00052

Lucetti, D.L.; Lucetti, E.C.P.; Bandeira, M.A.M.; Veras, H.N.H.; Silva, A.H.; Leal, L.K.A.M.; Lopes, A.A.; Alves, V.C.C.; Silva, G.S.; Brito, G.A.; Viana, G.B. 2010. Anti-inflammatory effects and possible mechanism of action of lupeol acetate isolated from Himatanthus drasticus (Mart.) Plumel. Journal of Inflammation (London) 7: 60. https://doi.org/10.1186/1476-9255-7-60

Malik, B.; Pirzadah, T.B.; Tahir, I.; Abdin, M.Z.; Rehman, U.I.R. 2016. Phytochemical studies on Cichorium intybus L. (CHICORY) from Kashmir Himalaya using GC-MS. Journal of Pharmacy Research 10(11): 715-726.

Masendra, M; Ashitani, T.; Takahashi, K.; Lukmandaru, G. 2018. Lipophilic extractives of the inner and outer bark from six different Pinus species grown in Indonesia. Journal Forestry Research (29):13291336. https://doi.org/10.1007/s11676-017-0545-x

Mutai, C.; Bii, C.; Vagias, C.; Abatis, D.; Roussis, V. 2009. Antimicrobial activity of Acacia mellifera extracts and lupane triterpenes. Journal of Ethnopharmacology 123: 143-148. https://doi.org/10.1016/j.jep.2009.02.007

Old, K.M.; Vercoe, T.K.; Floyd, R.B.; Wingfield, M.J.; Roux, J.; Neser, S. 2002. Acacia spp. FAO/IPGRI Technical Guidelines for the Safe Movement of Germplasm 20. https://www.bioversityinternational.org/fileadmin/_migrated/uploads/tx_news/Acacia_spp._829.pdf

Om, A.D.; Azizan, K.A.; Baharum, S.N. 2016. Phytochemical screening of ubi gadong (Dioscorea hipsida) by using GC-MS. Seminar dan konvensyen penyelidikan perikanan, Fri Batu Maung: Pulau Pinang. https://www.researchgate.net/publication/307510333

Pedemera, A.M.; Guardia, T.; Calderón, C.E.; Rotelli, A.E.; de la Rocha, N.E.; Saad, J.R.; Verrilli, M.A.; Aseff, S.G.; Pelzer, L.E. 2010. Anti-inflammatory effect of Acacia visco extracts in animal models. Inflammopharmacol 18: 253-260. https://doi.org/10.1007/s10787-009-0028-6

Prayitno, T.A.; Widyorini, R. 2016. The adhesion properties of wood preserved with natural preserva- 
tives. Wood Research 61(2): 197-204. http://www.woodresearch.sk/articles.php

Prayitno,T.A.; Widyorini, R.: Lukmandaru, G. 2017. Bonding ability of sengon wood treated with natural extracts. Wood Research 62(6):951-960. http://www.woodresearch.sk/articles.php

Ragasa, C. Y.; Guardamano, J.D.; Tan, M.C.S.; Ulep, R.A.; Altena, I.A.V. 2016. Chemical constituents of Dioscorea luzonensis Schauer .International Journal of Current Pharmaceutical Review and Research 7(4): 223-224.

Ramalakshmi, S.; Muthuchelian, K. 2011. Analysis of Bioactive constituents from the ethanolic leaf extract of Tabebuia rosea (Bertol.) DC by Gas Chromatography - Mass Spectrometry. International Journal of ChemTech Research 3(3): 1054-1059.

Saeed, M.A.; Sabir, A.W. 2002. Irritant potential of triterpenoids from Ficus carica leaves. Fitoterapia 73(5): 417-420. https://doi.org/10.1016/S0367-326X(02)00127-2

Savi, A.; Calegari, G.C.; Santos, V.A.Q.; Pereira, E.A.; Teixeira, S.D. 2018. Chemical characterization and antioxidant of polysaccharide extracted from Dioscorea Bulbifera. Journal of King Saud University - Science 32(1):636-642. https://doi.org/10.1016/j.jksus.2018.09.002

Se Golpayegani, A.; Thévenon. M.; Gril, J.; Masson, E.; Pourtahmasi, K. 2014. Toxicity potential in the extraneous compounds of white mulberry wood (Morus alba). Maderas-Cienc Tecnol 16(2): 227-238. https://doi.org/10.4067/S0718-221X2014005000018

Şen, S.; Yalcin, M.; Taşçığlu, C.; Özbayram, A.K. 2017. Larvicidal activities of some bark and wood extracts against wood-damaging insects. Maderas-Cienc Tecnol 19(3): 273-284. https://doi.org/10.4067/S0718-221X2017005000023

Singh, M.K.; Gidwani, B.; Gupta, A.; Dhongade, H.; Kaur, C.D.; Kashyap, P.P.; Tripathi, D.K. 2015. A review of the medicinal plants of genus Orthosiphon (Lamiaceae). Int J Biol Chem 9(6): 318-331. https://doi.org/10.3923/ijbc.2015.318.331

Sotannde, O.A.; Yager, G.O.; Zira. B.D.; Usman, A. 2011. Termiticidal effect of neem extracts on the wood of Khaya senegalensis. Research Journal of Forestry 5(3): 128-138. https://doi.org/10.3923/rjf.2011.128.138

Ssemaganda, I.E.; Mugabi, P.; Tumwebaze, S.B. 2011. Effectiveness of selected preservatives in protecting Ugandan grown Eucalyptus grandis wood against termite attack. Maderas-Cienc Tecnol 13(2): 135142. https://doi.org/10.4067/S0718-221X2011000200001

Subraya, C.K.; Harikiran; Gupta, D. 2012. Antioxidant and anti-inflammatory activity of Alstonia scholaris R.Br. stem bark extract. Free Radicals and Antioxidants 2(2):55-57. https://doi.org/10.5530/ax.2012.2.2.9

Surburg, H.; Panten, J. 2006. Common fragrance and flavor materials. WILEY-VCH Verlag GmbH \& Co. KGaA: Weinheim. https://b-ok.asia/book/1003351/5a42ec.

Syofuna, A.; Banana, A.Y.; Nakabonge, G. 2012. Efficiency of natural wood extractives as wood preservatives against termite attack. Maderas-Cienc Tecnol 14(2): 155-163. https://doi.org/10.4067/S0718-221X2012000200003

Tascioglu, C.; Yalcin, M.; Troya, T.; Sivrikaya, H. 2012. Termiticidal properties of some wood and bark extracts used as wood preservatives. Bioresources 7(3): 2960-2969. https://doi.org/10.15376/biores.7.3.2960-2969

USDA. 2016. Dr. Duke's phytochemical and ethnobotanical databases. http://phytochem.nal.usda.gov/ 